\title{
Viscosité d'une suspension de bactéries : des efforts individuels aux efforts collectifs
}

Harold Auradou$^{(1)}$ (auradou@fast.u-psud.fr), Carine Douarche(2) (carine.douarche@u-psud.fr), Adama Creppy $^{(1)}$, Hector Matias Lopez ${ }^{(1)}$ et Éric Clément ${ }^{(3)}$ (eric.clement@upmc.fr)

(1) Laboratoire FAST, Université Paris-Sud, CNRS UMR 7608, 91405 Orsay

(2) Laboratoire de Physique des Solides, Université Paris-Sud, CNRS UMR 8502, 91405 Orsay (3) Physique et Mécanique des Milieux Hétérogènes (ESPCl, Université P. et M. Curie, Université Paris Diderot, CNRS UMR 7636), 10 rue Vauquelin, 75005 Paris

\section{En 1906, Albert Einstein [1]}

a montré que l'ajout de particules sphériques solides dans un fluide augmente sa viscosité. En est-il encore ainsi quand les particules sont « actives » (c'est-à-dire motiles) ? Des expériences récentes avec des suspensions de bactéries Escherichia coli, qui sont de type " pousseur », démontrent un effet opposé : aux faibles taux de cisaillement, la viscosité macroscopique du liquide chute jusqu'à devenir nulle.

Ce phénomène vient essentiellement du fait que la puissance motrice des bactéries contribue

à la puissance mécanique

fournie au fluide pour assurer

son écoulement, cette contribution

ayant une valeur nette non nulle en raison de l'organisation de la nage des bactéries soit individuellement, soit collectivement.
La viscosité d'un liquide caractérise la puissance mécanique à fournir pour qu'il s'écoule ${ }^{(a)}$ : plus le fluide est visqueux, plus cette puissance est importante. Pour les liquides simples (eau, huiles de faible poids moléculaire...), la viscosité est indépendante du taux de cisaillement et ne dépend que de la température ; il en va autrement des liquides plus complexes (solutions de macromolécules, solutions concentrées de particules...), pour lesquels la viscosité dépend du taux de cisaillement et varie même souvent au cours du temps. Un cas particulièrement intéressant est celui des fluides " actifs " comme, par exemple, ceux contenant, en suspension, des bactéries dont le mouvement est autonome. En effet, la nage des bactéries peut modifier le bilan énergétique de l'écoulement et, donc, la viscosité apparente mesurée. De plus, selon le mode de nage (micro-organismes " pousseurs " ou " tireurs ", définis dans l'article d'introduction), cette influence sur la viscosité peut être très différente [2].

Ainsi, Sokolov et Aranson ont observé pour la première fois [3] une réduction de la viscosité en produisant des microécoulements à la surface d'une suspension de bactéries motiles de type "pousseurs". En créant de minuscules tourbillons et mesurant les temps de décroissance, ou en appliquant un couple à une particule magnétique et en mesurant la vitesse de rotation résultante du fluide, les auteurs ont montré qu'il est possible d'obtenir en présence de "pousseuses", une réduction de la viscosité d'un facteur 7 par rapport au même liquide sans bactéries ou contenant des bactéries non motiles. Une autre étude [4] sur des algues Chlamydomonas reinhardtii de type "tireurs " montre, au contraire, un accroissement de la viscosité avec la concentration par rapport au cas des algues non motiles.

La sensibilité et la précision des mesures de Sokolov et Aranson ne permettaient cependant pas de déterminer le rhéogramme des suspensions, c'est-à-dire la variation de la viscosité en fonction du taux de cisaillement $\dot{\gamma}_{\mathrm{M}}$. Elles ne permettaient pas, en particulier, d'explorer le domaine des très faibles taux de cisaillement. Pour un fluide qui contient des particules passives, cette gamme des faibles taux de cisaillement correspond à une viscosité constante, un " plateau ». Les études théoriques montrent que pour les fluides actifs ce plateau existe toujours, mais que sa valeur est différente : dans le cas de "pousseuses ", la viscosité est diminuée, alors que dans le cas de "tireuses » la viscosité est augmentée ; on parle alors de "plateau actif » [3].

Nous décrivons maintenant des expériences réalisées avec un rhéomètre de haute sensibilité, permettant d'obtenir de tels rhéogrammes. Ces mesures portent sur l'ensemble du volume du fluide et pas seulement au voisinage de la surface libre. Même si l'interprétation détaillée de telles mesures est toujours délicate, l'enjeu est ici de mesurer une annulation (voire un changement de signe) de la contrainte tangentielle et de déterminer dans quel domaine de taux de cisaillement elle se produit [5]. 


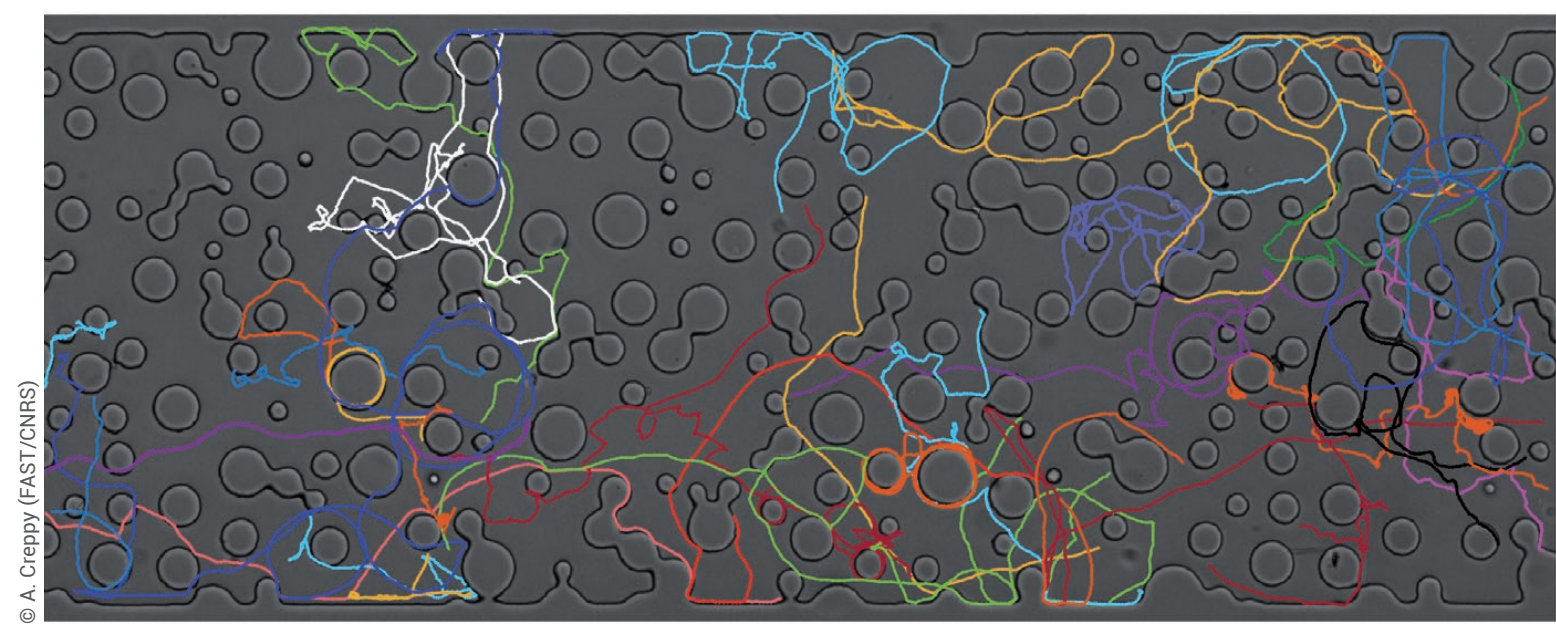

Superposition de trajectoires de bactéries $E$. coli (en couleurs) et d'une image de la cellule microfluidique (en niveaux de gris). Les durées des trajectoires sont supérieures à $10 \mathrm{~s}$. La cellule microfluidique est placée sous microscope (objectif x 10) et le champ de visualisation est de $1300 \mu \mathrm{m} \times 520 \mu \mathrm{m}$. La cellule contient des piliers de diamètre moyen $35 \mu \mathrm{m}$, disposés aléatoirement dans la cellule dont l'ouverture (l'épaisseur de liquide entre les parois dans la direction perpendiculaire au plan de l'image) est $100 \mu \mathrm{m}$. L'expérience est ici faite sans écoulement.

\section{Mesures de viscosité de suspensions de bactéries "pousseuses"}

On utilise un rhéomètre de Couette cylindrique (fig. 1a), où le cylindre extérieur tourne et le cylindre intérieur est suspendu à un fil de torsion de très faible diamètre et de grande longueur, qui assure une particulièrement bonne sensibilité (0,05 mPa.s). La position angulaire du cylindre intérieur est maintenue constante en appliquant, par une boucle de contreréaction, un couple égal et opposé à celui résultant de l'écoulement ; cette boucle fournit également un signal proportionnel au couple appliqué (et donc à la contrainte tangentielle $\Sigma$ sur le cylindre intérieur). Le mouvement relatif des deux cylindres impose un écoulement de cisaillement dans le volume de fluide ( $1 \mathrm{ml})$ situé entre ceux-ci (fig. 1b). Pour un taux de cisaillement moyen $\dot{\gamma}_{\mathrm{M}}$ sur l'ensemble de l'entrefer, on obtient alors, à partir de la contrainte mesurée, la viscosité effective moyenne de l'ensemble de la suspension; sa valeur combine les effets de la dissipation visqueuse et de la puissance mécanique

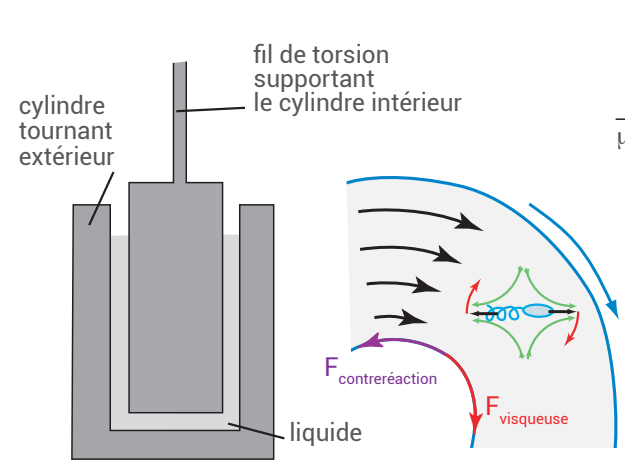

a

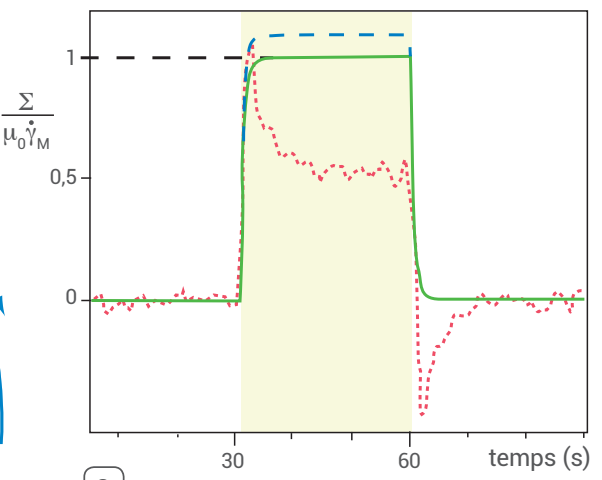

c

apportée par la nage des bactéries. Le fluide est une suspension de bactéries Escherichia coli de souche sauvage de type "pousseuses", en suspension dans une solution qui leur permet de rester motiles sans qu'elles se divisent pour que leur nombre reste constant.

Le cylindre extérieur est mis en rotation à vitesse constante pendant 30 secondes, puis arrêté. L'expérience est répétée pour différents taux de cisaillement moyens $\dot{\gamma}_{M}$ imposés et différentes solutions. La figure $1 \mathrm{c}$ montre, pour trois conditions différentes, les variations temporelles de la viscosité relative $\Sigma /\left(\mu_{0} \dot{\gamma}_{\mathrm{M}}\right)$ égale, par définition, au rapport $\mu_{\mathrm{r}}$ entre la viscosité effective de la suspension et celle $\mu_{0}$ du fluide porteur.

Pour un fluide sans bactéries (ligne continue verte), le signal mesuré suit instantanément la mise en rotation et la viscosité relative $\mu_{\mathrm{r}}$ reste constante et égale à 1 pendant toute la durée du créneau. Un comportement assez semblable est obtenu lorsqu'une suspension de bactéries est soumise à un taux de cisaillement élevé (tirets bleus). La principale différence avec la mesure en l'absence de bactéries se trouve dans la valeur du plateau atteinte, qui est légèrement supérieure à 1 . Cette augmentation est cohérente avec la théorie d'Einstein [1], qui prévoit une augmentation de la viscosité d'un fluide lorsque des particules y sont mises en suspension.

En revanche, pour des taux de cisaillement de l'ordre de la fréquence des changements de direction des bactéries (de l'ordre du hertz), la variation temporelle est bien différente (pointillés rouges). On observe

a) Schéma du rhéomètre de Couette.

b) Champ de vitesse du fluide entre les cylindres. Le mouvement d'une bactérie $E$. coli est représenté.

c) Variation temporelle de la viscosité relative $\mu_{r}=\Sigma / \mu_{0} \dot{\gamma}_{M}$ mesurée par le viscosimètre pendant une variation en créneau (durée de $30 \mathrm{~s}$ qui correspond à la zone jaune) de la vitesse de rotation sur des échantillons avec ou sans bactéries en suspension. $\Sigma$ est la contrainte tangentielle mesurée $\mu_{0}(=1,35 \pm 0,05 \mathrm{mPa} . \mathrm{s})$ est la viscosité du fluide porteur, et $\dot{\gamma}_{\mathrm{M}}$ le taux de cisaillement moyen. La ligne continue verte (resp. pointillée rouge) montre, pour un même taux de cisaillement $\dot{\gamma}_{M}=0,04 \mathrm{~s}^{-1}$, les mesures pour un fluide sans bactéries (resp. avec une fraction volumique $\phi=0,67 \%$ en bactéries). La ligne en tirets bleus montre la mesure sur la suspension avec bactéries, mais pour $\dot{\gamma}_{M}=63,9 \mathrm{~s}^{-1}$ 
$>>$

que $\mu_{\mathrm{r}}$ se stabilise plus lentement : $\mu_{\mathrm{r}}$ augmente lors de la mise en rotation jusqu'à atteindre la valeur mesurée à fort taux de cisaillement (tirets bleus sur la figure 1c), puis diminue et ne devient constante qu'après une dizaine de secondes à une valeur nettement inférieure à 1 : la viscosité effective est donc inférieure à celle du fluide sans bactéries. Le temps de relaxation reflète, quant à lui, la dynamique de l'organisation des bactéries sous écoulement. De même, lors de l'arrêt de la rotation, la viscosité effective (et donc la contrainte tangentielle mesurée) devient négative, puis relaxe vers zéro avec un temps caractéristique similaire à celui de la dynamique d'apparition.

Les rhéogrammes de la suspension (variation de $\mu_{\mathrm{r}}$ avec $\dot{\gamma}_{\mathrm{M}}$ en régime stationnaire) donnent une vue détaillée de ces régimes pour différentes fractions volumiques $\phi$ en bactéries (fig. 2).

On y retrouve, aux forts taux de cisaillement $\left(\dot{\gamma}_{M} \geq 3 \mathrm{~s}^{-1}\right)$, une viscosité relative presque indépendante de $\dot{\gamma}_{M}$ (" plateau supérieur ") et n'augmentant que légèrement avec $\phi$ en suivant la loi d'Einstein : on a le même comportement que celui d'une suspension de particules passives. Les bactéries sont toutes alignées avec l'écoulement. Par symétrie, la contribution hydrodynamique du contre-écoulement due à la nage s'annule.
En revanche, aux faibles taux de cisaillement $\left(\dot{\gamma}_{M} \leq 0,1 \mathrm{~s}^{-1}\right)$ pour lesquels la vitesse $\mathrm{du}$ fluide près du cylindre mobile est $\mathrm{du}$ même ordre que la vitesse de nage des bactéries $(\sim 20 \mu \mathrm{m} / \mathrm{s})$ ou inférieure, on atteint un "plateau actif " où la viscosité relative $\mu_{\mathrm{r}}$ est indépendante de $\dot{\gamma}_{\mathrm{M}}$; cette valeur « plateau » décroît fortement quand la concentration $\phi$ augmente.

La réduction de $\mu_{\mathrm{r}}$ est cohérente avec les résultats de Sokolov-Aranson et de Hatwalne sur les "pousseuses »; mais nos expériences montrent, en plus, que la réduction de viscosité ne se produit qu'aux faibles valeurs de $\dot{\gamma}_{M}$, alors qu'aux valeurs élevées, au contraire, le comportement est celui de particules inertes. Enfin, la variation de $\mu_{\mathrm{r}}$ avec $\dot{\gamma}_{\mathrm{M}}$ dépend fortement de la concentration en bactéries, suggérant l'apparition d'effets collectifs augmentant avec les interactions entre particules.

Cherchons maintenant jusqu'où peut aller cette réduction de viscosité relative...

\section{Toujours plus fort : la viscosité peut s'annuler}

La variation de $\mu_{\mathrm{r}}$ en fonction de $\phi$ sur le " plateau actif » est tracée sur la figure 3 pour des fractions volumiques allant jusqu'à $\phi \sim 2 \%(\sim 20$ milliards de bactéries dans l'entrefer). Pour les expériences du type de celles de la figure 2, réalisées dans une suspension de bactéries dépourvue d'oxygène (symboles $O$ dans la figure 3 ), $\mu_{\mathrm{r}}$ décroit d'abord linéairement avec $\phi$ puis atteint une limite de l'ordre de 0,2 , constante sur une large gamme de concentrations.

E. coli est une bactérie qui a la faculté de vivre avec ou sans oxygène. Lorsqu'elle est privée d'oxygène cependant, sa vitesse de nage est réduite. Les résultats dans le rhéomètre sont encore plus spectaculaires lorsque les bactéries n'ont pas encore consommé l'oxygène de la solution et que leur vitesse de nage est restée très rapide. Après une décroissance initiale linéaire avec $\phi$ plus rapide que sans oxygène, $\mu_{r}$ devient en effet nulle à l'erreur de mesure près et paraît même négative pour certains points (symboles $\bullet$ dans la figure 3 ). Plus aucune contrainte n'est alors détectée par le fil de torsion, comme si le fluide avait disparu. En fait, la contrainte sur le cylindre intérieur due aux écoulements induits par la nage des bactéries annule la composante visqueuse résultant du mouvement relatif des cylindres : en termes de bilan énergétique, l'apport de la nage des bactéries compense la dissipation visqueuse dans le fluide porteur et la dissipation apparente totale est nulle.

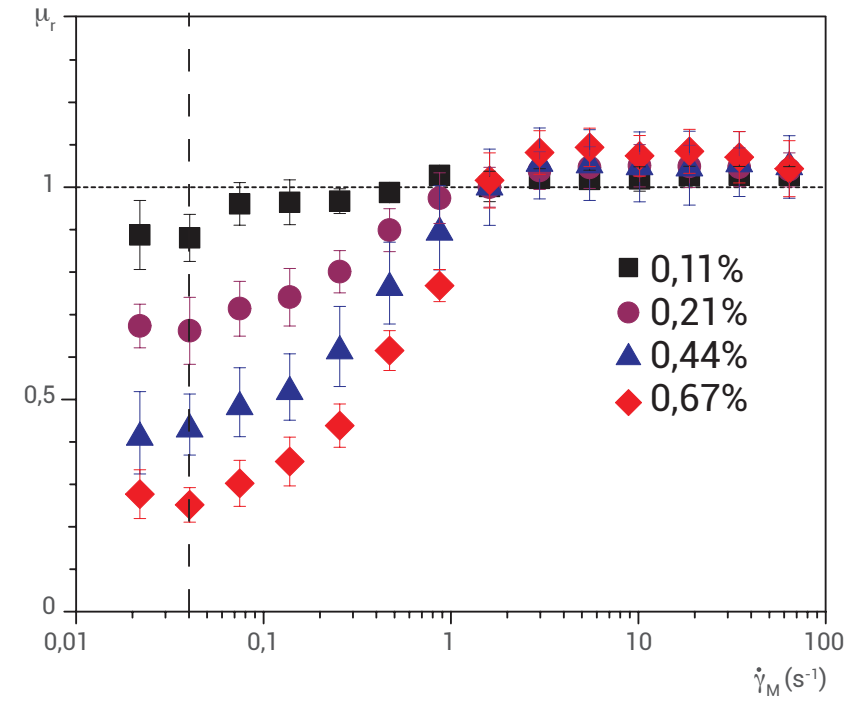

2. Variation de la viscosité relative avec le taux de cisaillement de suspensions de bactéries $E$. coli. Rhéogrammes $\left(\mu_{r}\right.$ sans unité $v s \dot{\gamma}_{M}$ en $\left.s^{-1}\right)$ mesurés à différentes fractions volumiques : $0,11 \% \leq \phi \leq 0,67 \%$ (soit entre 1 et 6,7 milliards de bactéries dans l'entrefer du rhéomètre).

La ligne verticale en tirets correspond au taux de cisaillement $\dot{\gamma}_{M}=0,04 \mathrm{~s}^{-1}$ repris sur la figure 3 .

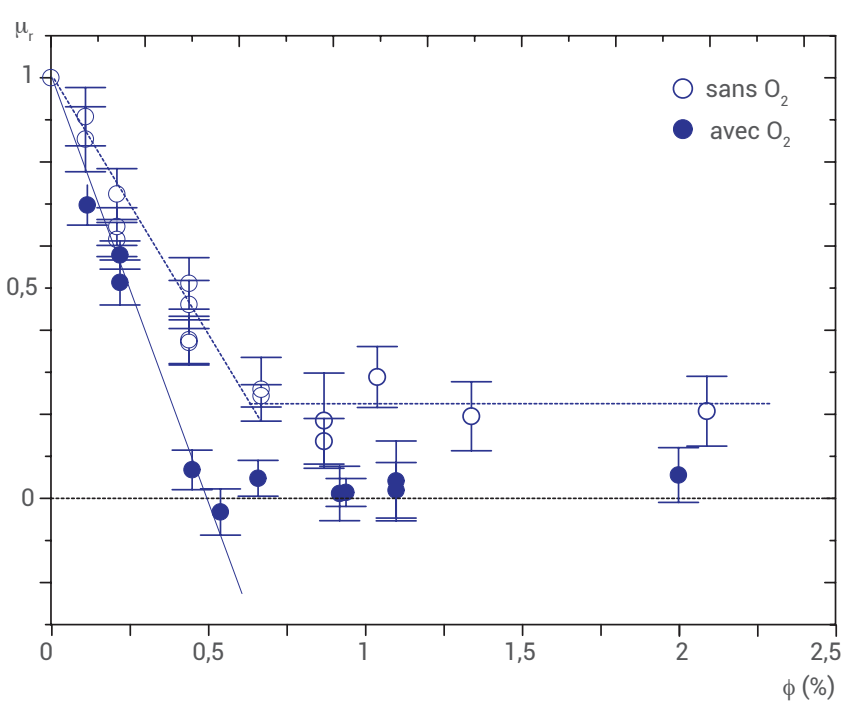

3. Variation de la viscosité relative $\mu_{r}$ en fonction de la fraction volumique $\phi$ des bactéries pour un taux de cisaillement $\dot{\gamma}_{M}=0,04 \mathrm{~s}^{-1}$. 0 : expériences sans oxygène ;

- : expériences avec oxygène.

La viscosité $\mu_{0}$ sans bactéries est de 1,35 $\pm 0.05 \mathrm{mPa}$.s. 


\section{Origine microscopique de la réduction de viscosité}

Comme l'ont montré Hatwalne et al. [2], l'existence d'une orientation préférentielle des bactéries induite par l'écoulement joue un rôle clé dans la diminution considérable de viscosité observée pour les bactéries de type «pousseur » comme E. coli. Les microorganismes s'alignent dans les écoulements comme les molécules d'un cristal liquide nématique. Ceci n'est possible que parce qu'elles ont une forme allongée ; l'écoulement de cisaillement n'aurait aucun effet si les bactéries étaient sphériques.

On explique cette orientation préférentielle par deux effets concurrents. D'une part, dans un écoulement de cisaillement, des particules allongées subissent un couple qui tend à les faire tourner (flèches rouges dans la figure 1b). D'autre part, ces bactéries peuvent modifier aléatoirement la direction de leur mouvement en désassemblant leurs flagelles de propulsion (voir l'introduction). Aux fortes valeurs de $\dot{\gamma}_{M}$, le premier effet est dominant et les bactéries tournent dans le plan perpendiculaire à l'axe des cylindres ; la vitesse de rotation est cependant variable et, en pratique, les bactéries sont pendant une grande fraction du temps approximativement parallèles à l'écoulement (fig. 4a). Dans ce cas, l'influence des écoulements produits par les bactéries (sur l'écoulement imposé) est en moyenne nulle. On a alors le même comportement rhéologique et la même viscosité que pour des colloïdes passifs de même géométrie, comme le confirment les mesures de viscosité.

Si $\dot{\gamma}_{M}=0$, les bactéries effectuant des mouvements d'orientation aléatoire, la moyenne des effets qu'elles induisent est alors nulle. Par contre, lorsque $\dot{\gamma}_{M}$ est faible mais non nul, un calcul complet montre que la combinaison des effets de réorientation aléatoire des bactéries et du couple provoqué par le cisaillement hydrodynamique conduit à une inclinaison moyenne non nulle des bactéries par rapport à la direction de l'écoulement imposé (fig. 4b) [6]. Si la vitesse de nage des bactéries est grande, la viscosité qui en résulte peut devenir inférieure à celle du fluide environnant. Plus la densité de bactéries est importante, plus la valeur de la viscosité diminue. Cet effet est observé pour les bactéries "pousseuses ». Pour des bactéries de type "tireuses ", le champ d'écoulement autour de la bactérie est inversé : l'effet de la nage accroît alors, au contraire, la viscosité du fluide.
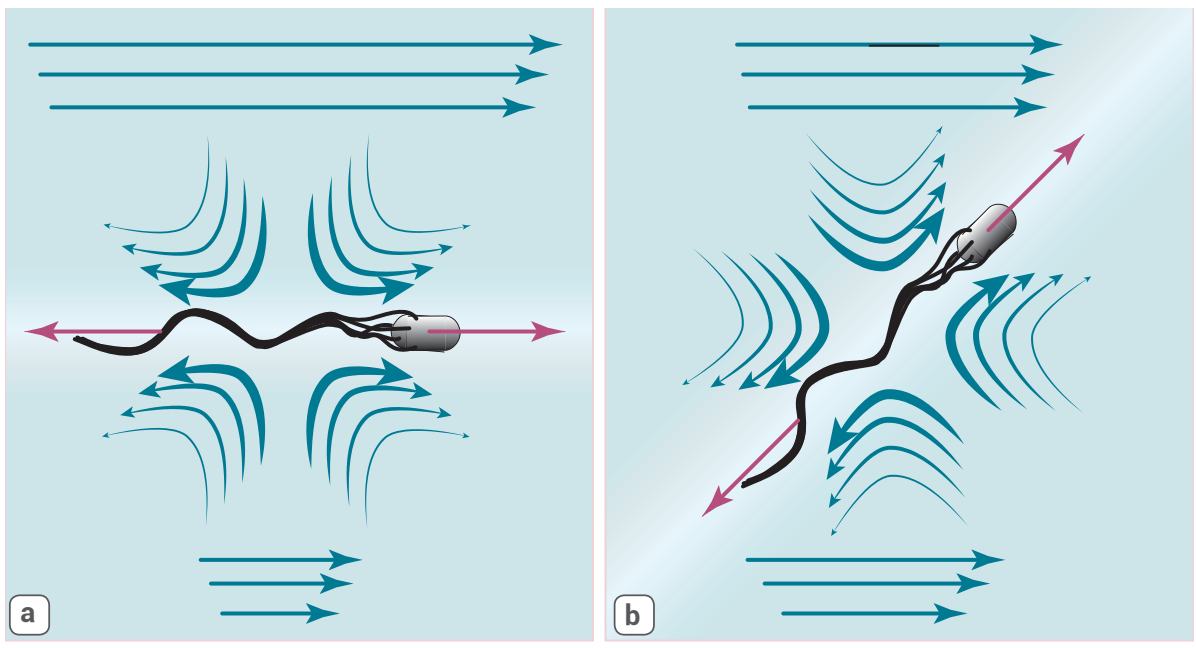

4. Influence des bactéries pousseuses sur l'écoulement d'un fluide. Orientation des bactéries (représentées en noir et gris) et champs de vitesse d'écoulement produits par les bactéries (lignes bleues fléchées courbes) et par l'écoulement de cisaillement imposé au fluide (flèches droites bleues). Les flèches droites rouges représentent les forces exercées par les bactéries sur le fluide, modélisées par un dipôle de forces.

(a) Forts taux de cisaillement.

(b) Faibles taux de cisaillement. La contribution de chaque bactérie est très faible mais, en additionnant celles des quelques milliards d'entre elles présentes dans l'entrefer, la réduction de viscosité est macroscopiquement mesurable.

\section{Conclusion}

Ainsi, la puissance mécanique fournie par la nage des bactéries " pousseuses " peut compenser complètement les pertes par dissipation visqueuse et conduire à une viscosité apparente nulle aux faibles taux de cisaillement. On peut donc, en principe, extraire de la puissance mécanique à l'échelle macroscopique à partir de l'activité bactérienne, en accord avec l'observation plus ancienne de la mise en rotation de microengrenages par des effets collectifs de mouvements bactériens. Des applications au mélange de fluides, aux petites échelles ou dans des dispositifs microfluidiques, pourraient être envisageables.

D'un point de vue plus fondamental, un écoulement totalement non dissipatif ne se rencontre qu'à basse température dans des systèmes quantiques où les phénomènes rencontrés, superfluidité et supraconductivité, sont bien évidemment très différents de ceux observés dans les suspensions de bactéries. Néanmoins, dans ces deux processus quantiques, l'intervention d'une fonction d'onde macroscopique de phase cohérente d'un atome à l'autre rappelle l'organisation de la nage des bactéries d'Escherichia coli, résultant de leurs interactions et compensant la dissipation visqueuse. Pour mieux comprendre ces phénomènes, des études statistiques de la dynamique collective de nage des bactéries seront nécessaires. (a) Dans un écoulement de cisaillement d'un fluide entre deux plans parallèles en mouvement relatif, la viscosité est le rapport entre la contrainte tangentielle $\Sigma$, définie par unité de surface des plans (aussi appelée " contrainte de cisaillement") et le gradient transverse de la vitesse du fluide (appelé dans l'article «taux de cisaillement " et noté $\dot{\gamma}_{\mathrm{M}}$ ). La viscosité a la dimension du produit d'une pression par un temps (mPa.s dans l'article) et le taux de cisaillement a la dimension d'une fréquence.

\section{Références}

1 A. Einstein, "Eine neue bestimmung der moleküldimensionen", Annalen der Physik, 324, 2 (1906) 289-306

2. Y. Hatwalne, S. Ramaswamy, M. Rao et R. A. Simha, "Rheology of active-particle suspensions", Phys. Rev. Lett. 92 (2004) 118101.

3. A. Sokolov et I. S. Aranson, "Reduction of viscosity in suspension of swimming bacteria?", Phys. Rev. Lett. 103 (2009) 148101

D. Saintillan, "The dilute rheology of swimming suspensions: a simple kinetic model", Exp. Mech. 5 (2010) 1275.

4 - S. Rafaï, L. Jibuti et P. Peyla, "Effective viscosity of microswimmer suspensions", Phys. Rev. Lett. 104 (2010) 098102.

5• H. M. Lopez, J. Gachelin, C. Douarche, H. Auradou et É. Clément, "Turning bacteria suspensions into superfluids", Phys. Rev. Lett. 115 (2015) 028301

6. R. Rusconi, J. S. Guasto et R. Stocker, "Bacterial Transport Suppressed by Fluid Shear", Nature Physics 10 (2014) 212-217. 